\title{
Molded Formulation Process
}

National Cancer Institute

\section{Source}

National Cancer Institute. Molded Formulation Process. NCI Thesaurus. Code C112974.

A process that forms a solid dosage unit by pouring or casting a melted agent into a prefabricated form. 